\title{
A new computational strategy for predicting essential genes
}

\author{
Jian Cheng ${ }^{1,2+}$, Wenwu $\mathrm{Wu}^{1,3+}$, Yinwen Zhang ${ }^{1,2}$, Xiangchen $\mathrm{Li}^{1,2}$, Xiaogian Jiang ${ }^{1,2}$, Gehong Wei, ${ }^{4,5^{*}}$ \\ and Shiheng Tao ${ }^{1,2,4^{*}}$
}

\begin{abstract}
Background: Determination of the minimum gene set for cellular life is one of the central goals in biology. Genome-wide essential gene identification has progressed rapidly in certain bacterial species; however, it remains difficult to achieve in most eukaryotic species. Several computational models have recently been developed to integrate gene features and used as alternatives to transfer gene essentiality annotations between organisms.

Results: We first collected features that were widely used by previous predictive models and assessed the relationships between gene features and gene essentiality using a stepwise regression model. We found two issues that could significantly reduce model accuracy: (i) the effect of multicollinearity among gene features and (ii) the diverse and even contrasting correlations between gene features and gene essentiality existing within and among different species. To address these issues, we developed a novel model called feature-based weighted Naive Bayes model (FWM), which is based on Naive Bayes classifiers, logistic regression, and genetic algorithm. The proposed model assesses features and filters out the effects of multicollinearity and diversity. The performance of FWM was compared with other popular models, such as support vector machine, Naïve Bayes model, and logistic regression model, by applying FWM to reciprocally predict essential genes among and within 21 species. Our results showed that FWM significantly improves the accuracy and robustness of essential gene prediction.
\end{abstract}

Conclusions: FWM can remarkably improve the accuracy of essential gene prediction and may be used as an alternative method for other classification work. This method can contribute substantially to the knowledge of the minimum gene sets required for living organisms and the discovery of new drug targets.

Keywords: Essential genes, Naïve Bayes, Support vector machine, Gene essentiality

\section{Background}

Essential genes, as a minimal gene subset in organisms, are required for survival, development, or fertility $[1,2]$. Therefore, the prediction and identification of such genes is not only interesting but also of theoretical and practical significance. Enhanced knowledge of essential genes promotes an understanding of the primary structure of the complex gene regulatory network in a cell [3-5] and helps elucidate the relationship between genotype and phenotype [6,7], identify human diseases [8],

\footnotetext{
* Correspondence: weigehong@nwsuaf.edu.cn; shihengt@nwsuaf.edu.cn tEqual contributors

${ }^{4}$ College of Life Science, Northwest A\&F University, Yangling 712100, Shaanxi, China

'College of Life Science, State Key Laboratory of Crop Stress Biology for Arid Areas, Northwest A\&F University, Yangling, Shaanxi, China

Full list of author information is available at the end of the article
}

discover potential drug targets in novel pathogens $[9,10]$, and re-engineer microorganisms $[11,12]$.

Two types of approaches are mainly used to predict and identify essential genes: experimental laboratory techniques and computational techniques. The former is randomly or systematically used to inactivate potential essential genes, and gene essentiality could be determined based on the living situation of the organism. General gene disruption strategies include single gene knockouts [13], conditional knockouts [14], RNA interference [15], and transposon mutagenesis [16]. Unfortunately, experimental techniques have significant drawbacks, such as long durations and high costs. In addition, the spectrum of gene essentiality varies under different growth conditions $[6,17]$.

Computational techniques have become popular over the past years for several reasons. First, known essential

\section{Biomed Central}


genes from dozens of microorganisms provide instructional and training materials. Second, the available genome sequences obtained by high-throughput sequencing provide unprecedented opportunities for investigating the minimal subset of genes in various organisms. Finally and most importantly, the development of bioinformatics tools improves our capability for exploring essential genes.

Several prediction models have been developed in silico to identify essential genes. Among these models, the simplest one is prediction of essential genes based on the known essentiality of homologous genes [18-21]. Although these prediction models show high confidence levels, they still have two limitations: first, the conserved orthologs between species only account for a small portion of the genome [22] and, second, the orthologs, especially in distantly related species, often show variations in gene regulations and functions $[6,23]$, which lead to potential diversity in gene essentiality. To circumvent these limitations, feature-based models have been constructed to distinguish essential genes from non-essential ones based on common or similar features among essential genes [24-28].

In previous models, feature selection was often based on significant correlations between gene essentiality and gene features or the significant distribution difference between essential and non-essential genes [29-31]. A common disadvantage of such selection method, however, is that feature-feature interactions and strong correlations among features are ignored [32]. Moreover, because of evolutionary divergence among species, the linkages between features and gene essentiality might have changed. For example, arguments on whether or not younger genes are less likely to be essential than older genes [33,34] or whether or not duplicate genes are less likely to be essential than singletons [34-36], demonstrate that gene essentiality associations with origin time and number of duplications are diverse among different species.

Aside from feature selection, machine learning algorithms have also been introduced into feature-based classification models to identify essential genes in many studies, such as Naïve Bayes [25], decision tree [26], and support vector machine (SVM) [27].

In the present study, we first collected 16 features (see feature abbreviations in Table 1) that were widely used in previous models, and demonstrated that the predictions exhibit at least two problems: (1) strong correlations among gene features and (2) different and even contrasting associations of gene features with gene essentiality among different species. We then presented a novel approach, the feature-based weighted Naïve Bayes model (FWM), which can address multicollinearity impacts among gene features and feature divergence between species. In the proposed model, prior information was
Table 1 Abbreviations and descriptions of selected features

\begin{tabular}{ll}
\hline Abbreviation & Description \\
\hline GO & Gene ontology \\
mE & mRNA expression level \\
mEF & mRNA express fluctuation \\
Age & Gene origin age \\
DoT & Gene domain type \\
DoC & Gene domain conservation \\
DC & Network topology feature, degree centrality \\
CCo & Network topology feature, clustering coefficient \\
CC & Network topology feature, closeness centrality \\
BC & Network topology feature, betweenness centrality \\
PL & Protein length \\
CAl & Codon adaptation index \\
NP & Number of paralogs for a target gene \\
NS & Number of species which have at least a homology \\
for a target gene & Number of essential homolog genes in other \\
sNH & species for a target gene \\
\hline
\end{tabular}

collected to determine the weight of each feature by logistic regression and genetic algorithm [37]. Afterward, essential genes in target organisms were predicted using a weighted Naïve Bayes (WNB) classifier [38]. We applied FWM to reciprocally predict essential genes between and within 21 species and compared its performance with those of other models including SVM, Naïve Bayes model (NBM), and logistic regression model (LRM). Results showed that FWM can significantly improve the accuracy and robustness of essential gene prediction. Finally, using stepwise discriminate analysis (SDA), we demonstrated why FWM outperforms these other classifiers.

\section{Results and discussion}

\section{Relationship of gene features and gene essentiality}

Selecting features associated with gene essentiality is fundamental to predict essential genes in feature-based models. However, because of the correlations between features, some features may actually share no or very few linkages with gene essentiality. Moreover, although feature linkages with gene essentiality exist, these linkages in different species are diverse or have contrasting effects.

To illustrate the possible consequences of different features in essential gene prediction, we investigate the linkages between gene essentiality and gene features in the Saccharomyces cerevisiae (SCE, Table 2A) and Escherichia coli $(E C O$, Table 2B) genomes, using the stepwise regression model (SRM) combined with forward selection 
Table 2 Linkages of features and gene essentiality in SCE (A) and ECO (B)

\begin{tabular}{|c|c|c|c|c|c|}
\hline \multicolumn{6}{|l|}{ A } \\
\hline Feature & Correlation $^{1}$ & P-value ${ }^{2}$ & True effect ${ }^{3}$ & P-value ${ }^{4}$ & R Square $^{5}$ \\
\hline DC & 0.256 & $3.7 \mathrm{E}-58$ & 0.157 & $1.6 \mathrm{E}-09$ & 0.065 \\
\hline $\mathrm{NEH}$ & 0.188 & $5.5 \mathrm{E}-32$ & 0.345 & $4.8 \mathrm{E}-61$ & 0.087 \\
\hline NP & -0.054 & 4.5E-04 & -0.186 & $2.5 \mathrm{E}-23$ & 0.118 \\
\hline NS & 0.001 & 0.47034 & -0.225 & $8.0 \mathrm{E}-24$ & 0.133 \\
\hline Age & -0.138 & $5.9 \mathrm{E}-18$ & -0.150 & $8.3 \mathrm{E}-15$ & 0.150 \\
\hline $\mathrm{CCO}$ & 0.166 & 4.0E-25 & 0.080 & $2.3 \mathrm{E}-06$ & 0.155 \\
\hline DoC & 0.132 & $1.9 \mathrm{E}-16$ & 0.058 & $2.5 \mathrm{E}-04$ & 0.157 \\
\hline $\mathrm{mEF}$ & -0.048 & 0.00166 & -0.044 & 0.01224 & 0.159 \\
\hline CAl & 0.043 & 0.00398 & -0.033 & 0.07042 & 0.160 \\
\hline$P L$ & 0.020 & 0.10449 & 0.022 & 0.14817 & 0.161 \\
\hline $\mathrm{CC}$ & 0.204 & 2.1E-37 & 0.021 & 0.40713 & 0.161 \\
\hline $\mathrm{mE}$ & 0.086 & $5.4 \mathrm{E}-08$ & -0.009 & 0.65639 & 0.161 \\
\hline \multicolumn{6}{|l|}{$B$} \\
\hline Feature & Correlation & P-value & True effects & P-value & R Square \\
\hline DC & 0.486 & $6 . E-228$ & 0.368 & $1.2 \mathrm{E}-38$ & 0.237 \\
\hline $\mathrm{NEH}$ & 0.478 & 2.E-219 & 0.359 & 2.E-110 & 0.350 \\
\hline $\mathrm{NNH}$ & -0.148 & 1.7E-20 & -0.327 & $3.4 \mathrm{E}-42$ & 0.382 \\
\hline NS & 0.283 & $6.7 \mathrm{E}-72$ & 0.147 & $7.8 \mathrm{E}-11$ & 0.390 \\
\hline CC & 0.314 & $6.0 \mathrm{E}-89$ & -0.115 & $2.4 \mathrm{E}-06$ & 0.396 \\
\hline NP & -0.151 & $2.9 \mathrm{E}-21$ & 0.085 & $1.3 \mathrm{E}-05$ & 0.399 \\
\hline $\mathrm{mEF}$ & 0.147 & 2.7E-20 & -0.058 & $6.9 \mathrm{E}-05$ & 0.401 \\
\hline $\mathrm{CAl}$ & 0.302 & 3.7E-82 & 0.024 & 0.11874 & 0.402 \\
\hline $\mathrm{mE}$ & 0.266 & $1.4 \mathrm{E}-63$ & 0.036 & 0.02500 & 0.402 \\
\hline$P L$ & 0.038 & 0.00940 & 0.024 & 0.07566 & 0.403 \\
\hline DoC & 0.106 & $1.8 \mathrm{E}-11$ & 0.013 & 0.32555 & 0.403 \\
\hline $\mathrm{CCO}$ & 0.250 & $3.0 \mathrm{E}-57$ & 0.004 & 0.79730 & 0.403 \\
\hline
\end{tabular}

Note: The features from top to bottom in Column 1 are shown in accordance with the sequence in which they are added to the SRM. Data for gene essentiality in SCE and ECO originate from the relative growth rates of single-gene deletion yeast strains in nutrition-rich YPD medium [43] and E. coli chromosome (PEC) database profiling [44], respectively. Pearson correlations ${ }^{1}$ between gene essentiality and features are shown with a corresponding P-value ${ }^{2}$. True effect ${ }^{3}$ refers to the standardized regression coefficient in the model and it is presented with a related P-value ${ }^{4}$. R Square ${ }^{5}$ represents the ratio of the variance predicted by the SRM and the variance for gene essentiality.

[39-41]. At the beginning of the experiment, no features are considered in the model. A feature that mostly improves the model is added, and this process is repeated until all features are included in the model. The first column of Table 2 shows the results of the sequential addition of features into the SRM. Among the 12 features (Table 2A), the feature DC is the most important factor that explains the variation (6.5\%) of gene essentiality. Some close neighboring features (e.g., NEH and NP) show statistical significance in terms of both correlations and true effects (i.e., standardized regression coefficients) with gene essentiality in the model. The last selected features (i.e., CAI, CC, and $\mathrm{mE}$ ) also show statistical significance in linkage with gene essentiality; however, their true effects are detected without statistical significance (P-value $>$ $0.05)$. This result may be explained by the fact that $C C$ is highly correlated with DC $(r=0.765, \mathrm{P}$-value $<0.01)$, and $\mathrm{DC}$ has been selected as the first feature in the model that has diminished the effects of $\mathrm{CC}$. One reason that may explain the lack of significant true effects exerted by CAI and $\mathrm{mE}$ is that both features have significant correlations with DC $(r=0.298, r=0.393$, and both P-values $<0.01)$ and CC (data not shown). Another explanation is that some essential genes show low expression levels. For example, the genes whose products are located in nuclear part (GO: 0044428) are overrepresented among the essential genes with lower expression levels. In addition, some transcription factors and centromere-associated proteins are only required in small amounts; however, these substances may be expressed constitutively and indispensably [42]. A similar pattern is observed during $E C O$ analysis; however, compared with the $S C E$ genome (Table 2A), the same features (e.g., NS) often show distinct effects on gene essentiality in the SRM (Table 2B). Most features in SCE contribute much less to gene essentiality than those in $E C O$. The genes in $S C E$ are thus postulated to have more complicated and diverse functions than those in $E C O$, and the essentiality of these genes must be explained by a larger variety of features, which is expectedly in agreement with that eukaryotes are more complex than prokaryotes.

If excessive features that contributed less to the model were selected, the process would inevitably lead to a complex and inefficient regression model. Besides, the same feature can result in different or opposite effects in different species, (e.g., NS has opposite effects in ECO and $S C E$ ). Therefore, selection of improper or excessive features may lead to redundancy and decrease the accuracy of the essential gene prediction model. These effects contradict the original goal of the essentiality analysis. In the current study, to overcome the deficiency in essential gene prediction, we developed a new method called FWM.

\section{FWM construction}

Among the various classification methods, the Naïve Bayes classifier [45] is a simple, fast, and efficient algorithm. Thus, Naïve Bayes classifiers are widely used in identifying essential genes, disease genes, and housekeeping genes $[25,28,46-48]$. In the current study, we developed another method called FWM (Figure 1A) that effectively addresses the effects of multicollinearity among gene features in NBM and overcomes the disadvantages of training and prediction sets with equal global feature score (GFS, see Appendix). 


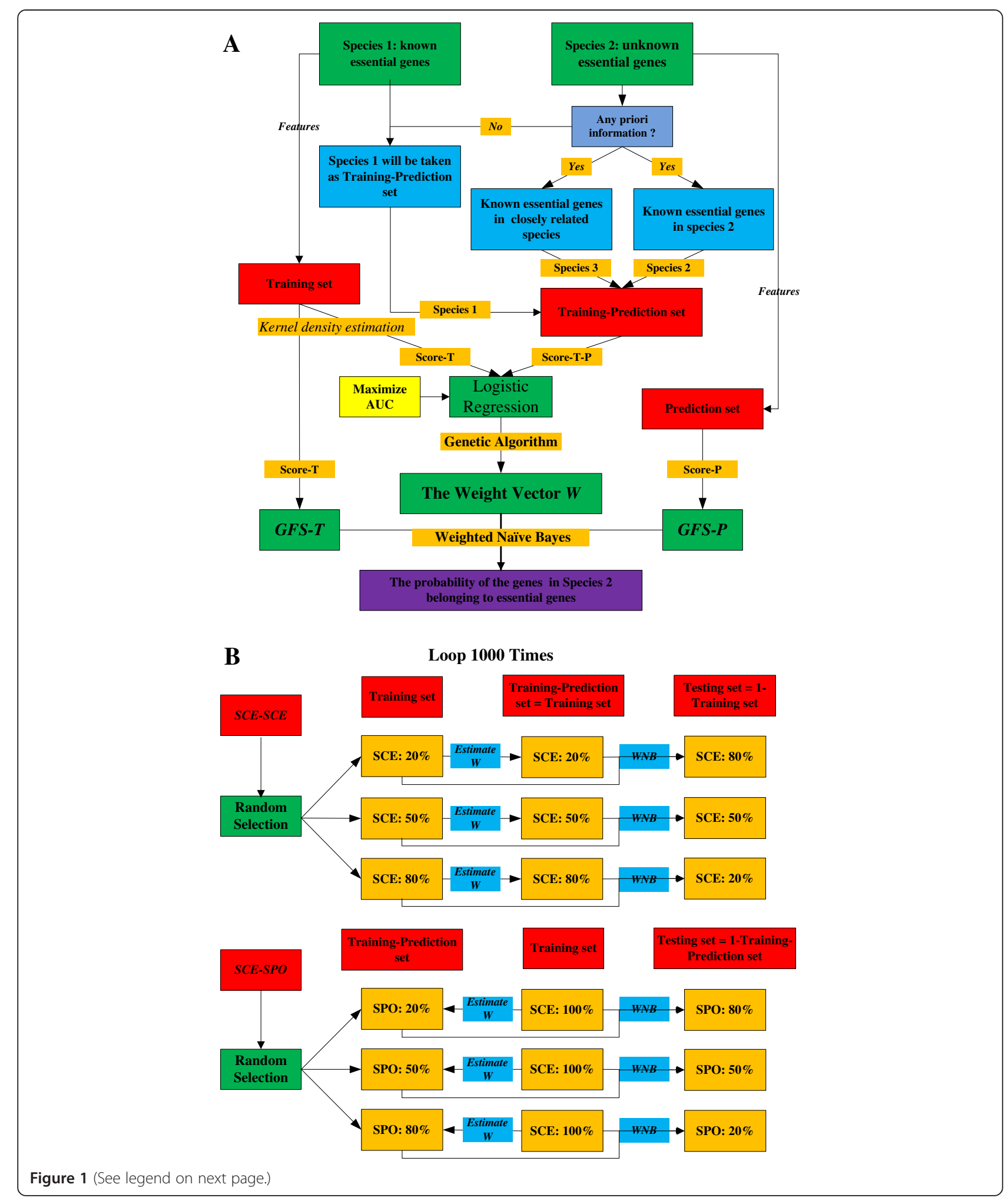


(See figure on previous page.)

Figure 1 Flow chart for constructing FWM and assessing its performance in predicting essential genes between and within species. (A) FWM construction. During essential gene prediction from species 1 to species 2 , the goal of FWM is to calculate the score vector $S_{i}$ and the weighted coefficient vector $W$. To calculate $S_{\text {i, }}$ we mainly employ kernel density estimation (KDE) combined with Naïve Bayes estimation (see Methods). When calculating $W$, we first collect prior information (e.g., known essential genes in species 2 or from a closely related species); this information is used as training-prediction dataset to assess $W$ in combination with the training set. Finally, we calculate the posterior probability of the genes in species 2 belonging to essential genes based on the weighted Naïve Bayes (WNB) method. (B) FWM performance for predicting essential genes between and within species. To assess the performance of FWM within species (e.g., SCE-SCE or SPO-SPO), 20\%, 50\%, and 80\% of the whole genes were randomly selected as the training set, respectively, and the rest as testing set. We used the training set itself as a training-prediction set to calculate weights; the AUC score for the testing set was then calculated through the WNB method. Finally, the process was replicated 1,000 times to obtain the corresponding AUC distributions. To predict essential genes between species (e.g., SCE-SPO or SPO-SCE), all of the genes in SCE (or SPO) were selected as the training set, $20 \%$ (or 50\%, 80\%) of the SPO (or SCE) genes were randomly selected as the training-prediction set, and the rest of the genes were designated as the testing set. Similar to the comparison within species, AUC distributions were obtained by replicating the process 1,000 times.

The basic FWM formula in the present study is described below (see inference in Appendix). For one gene $g_{i}$ $\left(g_{i} \in G, i=1,2, \cdots, m\right)$ with a feature vector $X_{i}$, the probability of the gene belonging to the class $E$ ( $E=$ essential) is:

$$
P\left(g_{i} \in E \mid X_{i}\right)=\frac{1}{1+e^{-S_{i} \cdot W}}
$$

where $W$ is the weight vector indicating the extent of contribution of the features to gene essentiality and $S_{i}$ is the feature score vector corresponding to logarithmic likelihood ratio: $S_{i}=\log \frac{P\left(X_{i} \mid g_{i} \in E\right)}{P\left(X_{i} \mid g_{i} \in \bar{E}\right)}$.

The key FWM procedure is the evaluation of $S_{i}$ and $W$. For $S_{i}$ calculation, our selected features are divided into continuous type (e.g., protein length) and non-continuous type (e.g., domain type); we then employ kernel density estimation [49] and Bayes estimation to calculate the $S_{i}$ of these two types of features (see Methods).

To evaluate $W$, we need a $W$ that can reflect the true contribution of the features in the target species. Therefore, we first determine some prior information based on a known essential gene set, which is preferably from the target species or a species that is closely related to the target species. Note that we define the known essential gene set as the training-prediction set used as a dependent variable to help evaluate $W$. If we cannot obtain any prior information, the training set is also used as an alternative training-prediction set to calculate $W$. According to formula (1), we obtain $\frac{S_{i} \times W=\log P\left(g_{i} \in E \mid X_{i}\right)}{1-P\left(g_{i} \in E \mid X_{i}\right)}$. We then imitate the estimation of logistic regression coefficients to calculate $W$. To obtain high essential gene prediction accuracy, we estimate the parameter $W=\operatorname{argmax}\{A U C[P P(W), G E]\}$ using genetic algorithm. Here, $P P(W)$ represents the posterior probability vector calculated by formula (1), $G E$ represents the true gene essentiality determined from the trainingprediction set, and the AUC (area under curve) score is calculated from $P P(W)$ and $G E$. Finally, we calculate the posterior probability of the genes in Species 2 (the target species whose essential genes need to be predicted) based on the WNB method again using formula (1).

\section{FWM accuracy, stability, and adaptability}

Because FWM is developed from NBM, we first compared the predictive performances of FWM and NBM within and between species. Two eukaryotic species (SCE and Schizosaccharomyces pombe (SPO)) that have wellcharacterized essential genes were used as either training sets or testing sets (Figure 1B).

To investigate the accuracy and stability of FWM, 20\% of the SCE genes were randomly selected as both the training set and the training-prediction set to help calculate $W$; the rest of the genes were used as the testing set. FWM and NBM were then used to predict and calculate AUC scores, respectively. The simulation was replicated 1,000 times (randomly selected training set and testing set), and two corresponding AUC distributions were obtained (Figure 2A). Similarly, 50\% and $80 \%$ of the genes in $S C E$ were respectively randomly selected and simulated with 1,000 replications to obtain the AUC distributions (Figures $2 \mathrm{C}$ and $\mathrm{E}$ ). By comparing the AUC distributions obtained by FWM and NBM, we found that the mean values from FWM were significantly higher than from NBM (T-test and P-value $<1 \mathrm{e}-100$; Figure 2A, C and E). This finding demonstrates that the results within species predicted by FWM are more accurate than those predicted by NBM. Similar results were obtained in SPO (Additional file 1: Figure S1-A, S1-C and S1-E).

To assess the adaptability of FWM between different species, we adopted the strategy to predict essential genes in SPO based on training dataset from SCE. First, 20\%, $50 \%$, and $80 \%$ of the SPO genes were randomly selected as training-prediction sets (the rest of the genes were used as the testing set), and the corresponding weight vector $W$ was obtained. We then predicted the remaining set of $S P O$ genes using $W$ and the training set from SCE. Finally, we obtained the AUC distributions with 1,000 replicated 

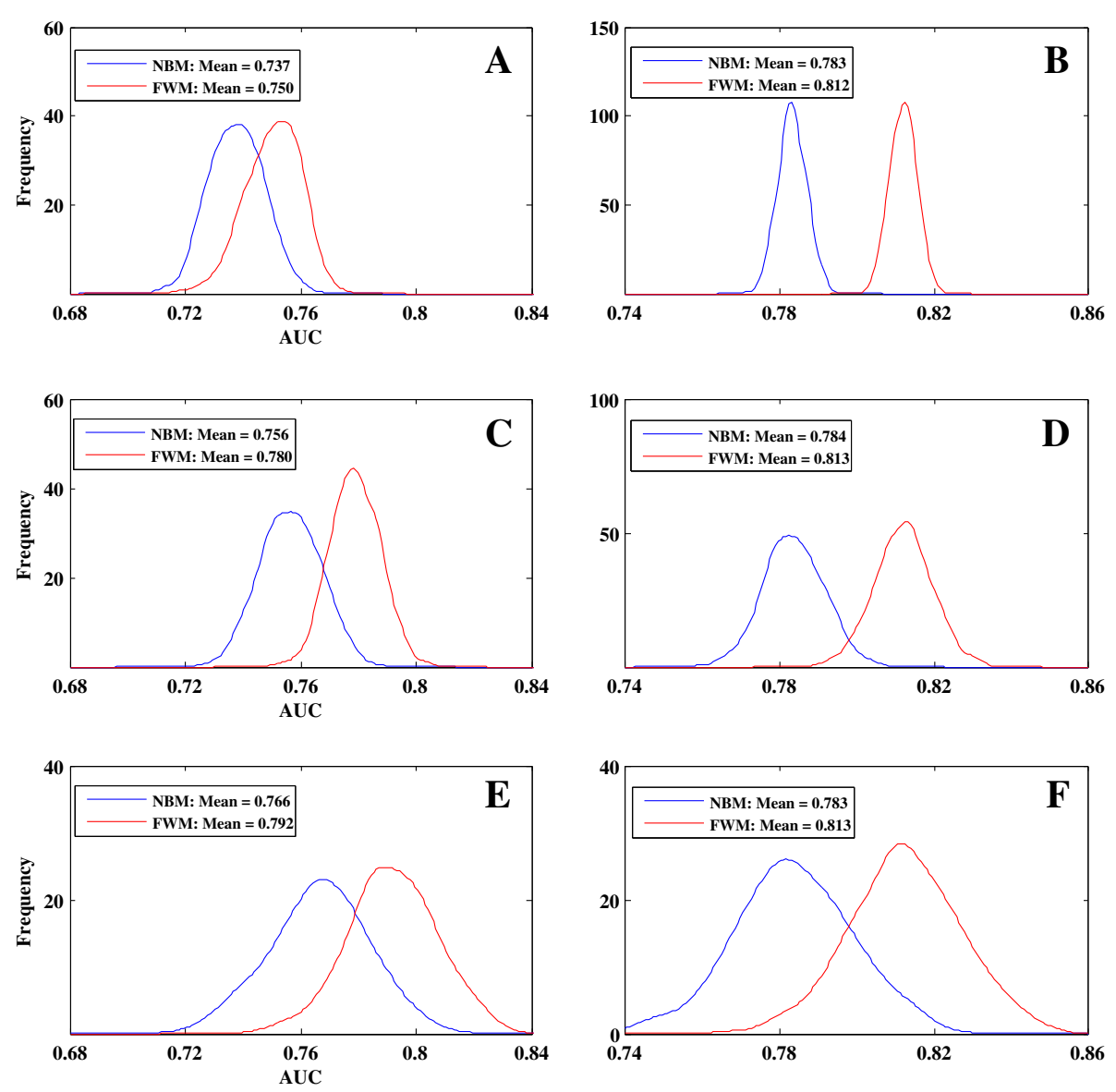

Figure 2 Essential gene prediction within and between species by NBM and FWM. A, C, and E show the AUC distributions within species (SCE-SCE), which are generated by randomly selecting 20\% (A), 50\% (C), and $80 \%$ (E) of the SCE genes as training data. B, D, and $\mathbf{F}$ show the AUC distributions between species (SCE-SPO), which are generated by randomly selecting 20\% (B), 50\% (D), and $80 \%$ (F) of the SPO genes as a training-prediction set to estimate the weight vector $W$. Blue and red lines represent the distributions obtained by NBM and FWM, respectively.

simulations. The results were shown in Figures 2B (20\%), $2 \mathrm{D}(50 \%)$, and $2 \mathrm{~F}(80 \%)$. A similar analysis was performed in the prediction from SPO to SCE (Additional file 1: Figures S1-B, S1-D, and S1-F). Consistent with the results of prediction within species, FWM showed better performance than NBM for predictions between species. In addition, while obtaining an accurate vector $W$, FWM easily reaches a saturation point when some prior information is supplied.

\section{Comparison of FWM with LRM, NBM, and SVM}

We applied FWM to 21 species (including 19 bacteria and two fungi; listed in Additional file 2: Table S1) to illustrate: (1) the validity of FWM predictions of essential genes in diverse species and (2) the advantages of FWM over other methods. The genes in the 21 species were taken in turns to use as training and testing sets. The process yielded a $21 \times 21$ AUC matrix represented as $M=\left(m_{i j}\right), i, j=1,2, \ldots$,
21 , where $m_{i j}$ indicates the AUC score obtained with $i$ th species as training set and $j$ th species as testing set.

The accuracy of FWM prediction was compared with three other classifiers: LRM, NBM, and SVM. Each of these classifiers yielded a $21 \times 21$ matrix with a total of 441 AUC scores (Additional file 2: Table S2) independently. Afterward, we sorted the AUC scores of variable $m_{i j}$ produced by the four approaches (Figure 3, see details in Additional file 2: Table S3). 61.7\% (272/441) AUC scores produced by FWM were ranked in first tier (which represented the AUC score is the maximum among the quadruple AUC scores generated by the four methods) and only one was located in the fourth tier (the AUC score is the minimum among the quadruple AUC scores). Evidently, FWM significantly outperformed the other three methods (P-value $<1 \mathrm{e}-53$ ). By the way, the performance of SVM was slightly but not significantly better than that of NBM (P-value = 0.343). Although the performance of LRM was the worst among the four approaches studied, 


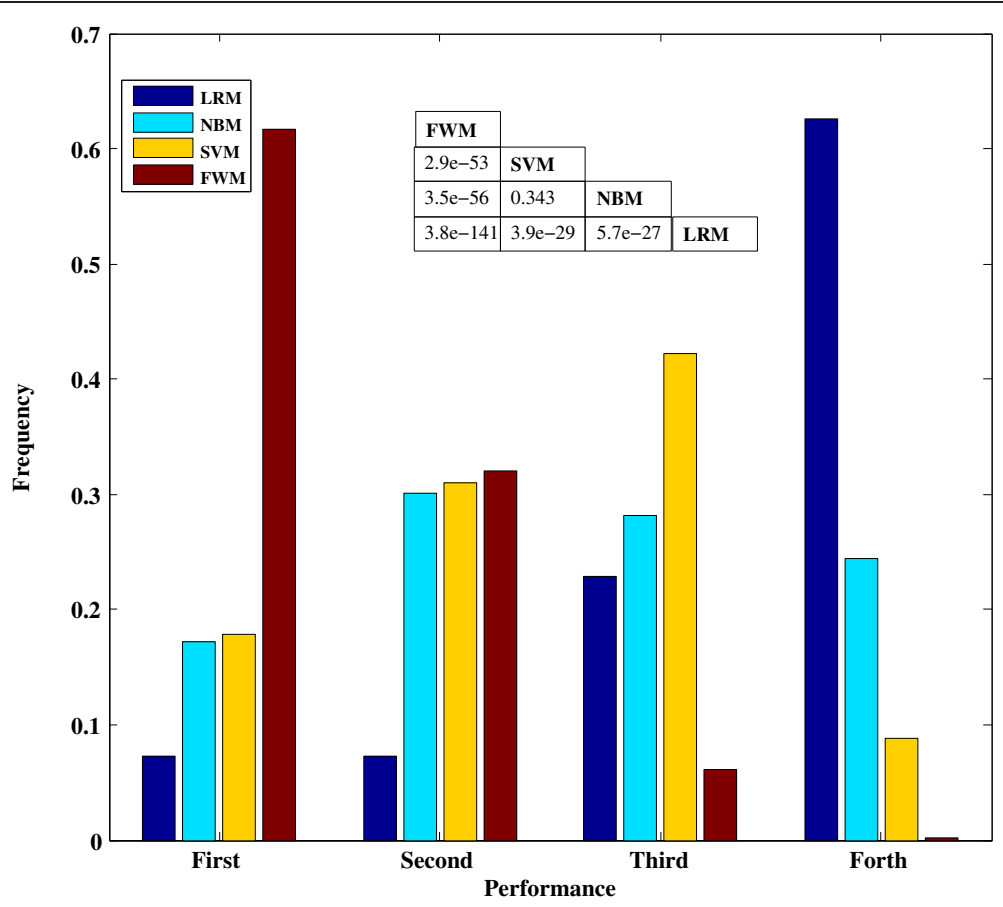

Figure 3 Comparison of FWM with LRM, NBM, and SVM. Four AUC matrices among the 21 species are produced using the four methods. AUC scores $\left(m_{i j}\right)$ in the same position of the four matrices are then sorted and replaced with markers (first with the maximum AUC score, followed by the second and third, and, finally, fourth with the minimum score) for the four methods. By calculating the frequency of the ranking list (i.e., first, second, third, and forth) in the four matrices, performance distributions for the four methods were generated. The AUC score with ranking the first and second can be classified as high-quality performance, while third and fourth can be classified as low-quality performance. Significant differences are tested by Fisher's exact test, and the results are shown in the lower triangular table.

this model showed strong overfitting, which can lead to better performance during cross-validation within species than NBM and SVM. Anyhow, our method is substantially superior to LRM, NBM, and SVM for predicting essential genes.

\section{Why does our approach perform better prediction effects?}

To explain why FWM outperforms the other classifiers, we employed SDA combined with forward selection [50] to investigate AUC variations; FWM and NBM were used as discriminate models. During the repeating selection process, the features that could improve AUC score the most in the classifiers were selected one by one, until all of the features were included in the model. Four microbes with well-characterized essential genes were used in our analysis (Figure 4), including two bacteria (i.e., ECO and Streptococcus sanguinis (SSA)) and two fungi (i.e., $S C E$ and $S P O)$. The labels on the $\mathrm{X}$-axis from left to right in Figure 4 indicated the order of the features selected into the model one by one. During analysis, the following features showed the best performance: $\mathrm{CC}$ in $S C E-E C O$, NEH in ECO-SSA and SSA-SPO, and DoT in $S P O-S C E$. The best performance of CC demonstrated that essential genes tend to play topologically more important roles in protein interaction networks than nonessential genes. SCE and ECO had more complete and available interaction data than the other organisms; thus, $\mathrm{CC}$ neither performs the best among the three other predictions. The best performance of $\mathrm{NEH}$ showed that orthologous gene essentiality is conserved across organisms. DoT had the best performance in SPO-SCE prediction because, relative to that in bacteria, gene essentiality is more conserved through the function of protein domains or domain combinations rather than through the conservation of the entire genes in fungi [28].

Although the feature GO has a better effect than other features in the prediction (see Additional file 2: Table S4), most genes with known GO annotations have only been recorded in well-studied model species but have not been investigated in the non-model organisms. Thus, selecting $\mathrm{GO}$ as the feature to predict essential genes in a nonmodel or a new sequenced organism is inappropriate. Values close to the $\mathrm{X}$-axis (Figure 4) indicate the singular prediction effect of the corresponding feature. Except for the first feature, the selection order of other features into the prediction model was based on the diminished effect from the previous selected features. For example, in the 

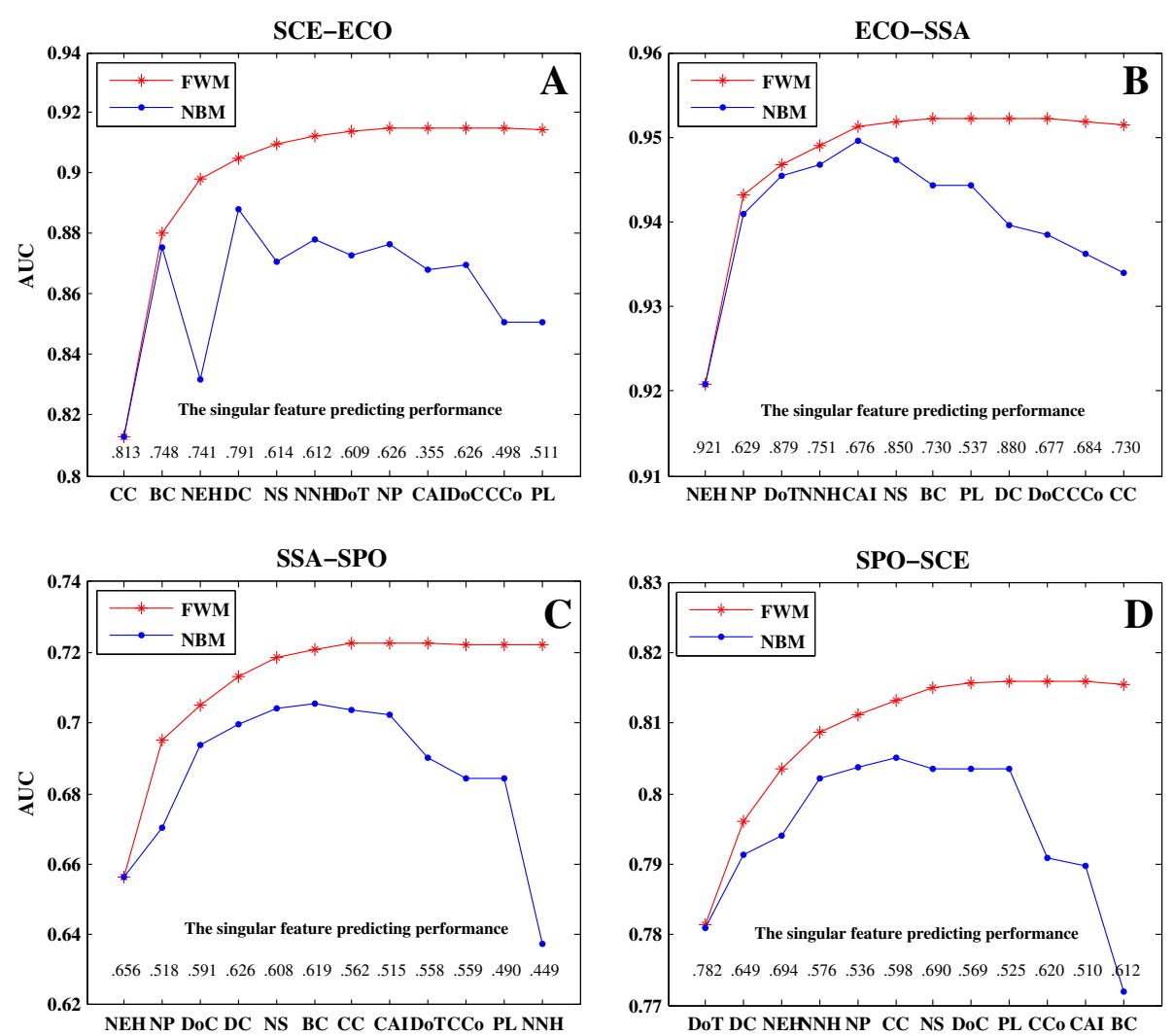

Figure 4 Comparison of FWM and NBM in stepwise discriminant. Examples of SCE-ECO (A), ECO-SSA (B), SSA-SPO (C), and SPO-SCE (D) are plotted. The labels on the $X$-axis from the left to right indicate the order of the features selected into the model according to their prediction effects. The values above the X-axis represent the singular prediction effect of the corresponding feature. FWM indicates feature-based weighted Naïve Bayes model and NBM indicates Naïve Bayes model.

prediction from $S P O$ to $S C E$, the AUC score generated by a single NS feature is 0.69 , which ranks third among all of the features; but this feature was selected as the seventh feature in SDA because of the partial replacement of NS effects by previously selected features.

FWM performed better than NBM in all cases. The prediction accuracy reached a saturation point when some key features were selected into the model. The NBM classifier substantially reduced the prediction accuracy at the end of prediction, whereas FWM provided redundant features with small weights to avoid such a problem (see Additional file 2: Table S5) and showed slow changes in prediction accuracy with addition of features.

In Figure 5, we compared receiver operating characteristic (ROC) curves generated by FWM and NBM in four microbes. FWM consistently showed a significant higher true positive rate (TPR) and a significantly lower false positive rate than NBM in all four predictions, except for the location of ROC curves at approximately 0 or 1 in the $\mathrm{X}$-axis. The increases in AUC based on FWM relative to NBM in SCE-ECO, ECO-SSA, SSA-SPO, and SPO-SCE are $0.064,0.018,0.085$, and 0.044 , respectively. AUC score also indicated the average TPR in all threshold values [51]; thus, our FWM could improve prediction accuracy at least from $2 \%$ to $9 \%$. In general, FWM provides a more effective way of integrating features associated with gene essentiality, and overcomes the impact of multicollinearity among features. Therefore, FWM presents the advantages of increased adaptability and reliability for essential gene prediction.

\section{Conclusions}

Selecting features associated with gene essentiality is necessary to identify essential genes through machine learning approaches. However, current studies often neglect the phenomenon of multicollinearity among these features, and the same feature may result in different and even contrasting effects among species. Selecting such features makes the prediction model cumbersome, does not improve prediction precision, but contrarily, may decrease the accuracy of essential gene prediction. In other words, selecting more features does not mean better prediction results. 

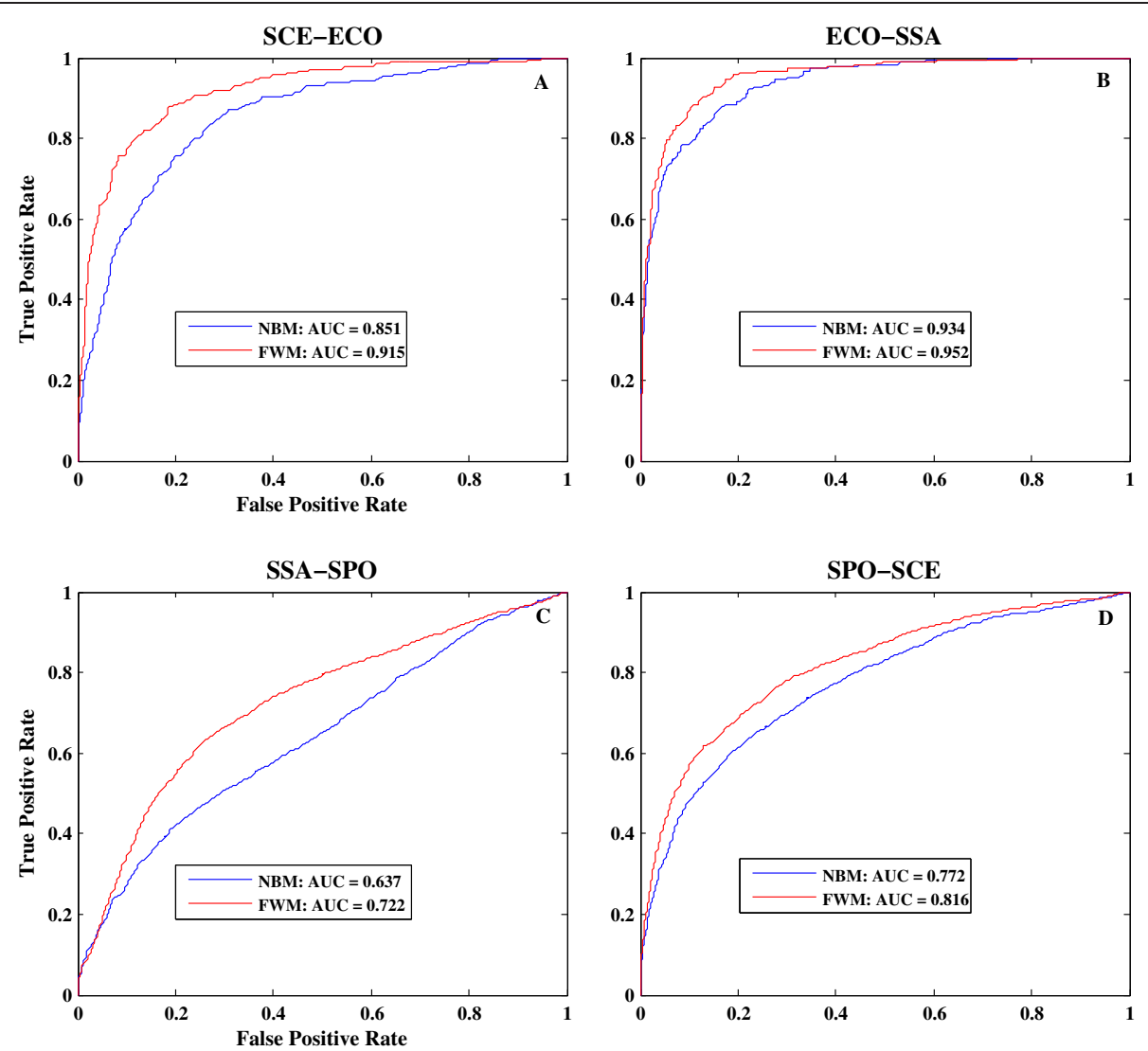

Figure 5 Comparison of ROC curves between FWM and NBM. Examples of SCE-ECO (A), ECO-SSA (B), SSA-SPO (C), and SPO-SCE (D) are plotted. TPR (sensitivity) is plotted on the Y-axis and FPR (1-Specificity) is plotted on the X-axis with threshold values from 0 to 1 . Blue lines represent ROC curves generated by NBM and red lines represent ROC curves generated by FWM. FWM indicates feature-based weighted Naïve Bayes model, NBM indicates Naïve Bayes model, and AUC indicates area under curve.

To address these problems, we built FWM by improving the Naïve Bayes classifier. This new model assigns a corresponding weight for each feature based on its real contribution to the model, and importantly, this weight can be changed depending on the specific target organisms. FWM is able to effectively alleviate the effects of both multicollinearity among features and the complex relationship between features and gene essentiality in different organisms. In summary, FWM is able to improve predictive accuracy compared with other methods (e.g., NBM, LRM, and SVM).

$\mathrm{Xu}$ et al. [52] revealed that essential genes are associated with only three basic categories of essential functions or processes in organisms: cell envelope maintenance, energy production, and genetic information processing. Nevertheless, the genes engaged in essential functions may yield a conditional essential gene during evolution [3,7]. Others may be compensated by a duplicate or buffered by some new metabolic network flux reorganization that results in the transformation of essentiality in the original reaction or path. Besides, because of changes in the external environment or evolution from lower to higher organisms, many new essential functions and metabolic processes can emerge. Thus, gene essentiality constantly changes over time and more efforts are needed to completely understand the minimal requirements for cellular life. In the current study, we presented a theoretical frame and a practical strategy to predict mass genomewide essential genes. Our method reduced the burden of systematic understanding of the minimal requirements for cellular life and can help identify potential drug targets in novel pathogens.

\section{Methods}

Essential gene and gene sequences

The essential genes of 21 species (see the species in Additional file 2: Table S1 and the collected essential gene in Additional file 3) were obtained from relevant studies, as well as the Online Gene Essentiality Database (OGEE) [53] and Database of Essential Genes (DEG) [54]. The cDNA and protein sequences of the 21 species were downloaded from the NCBI server (ftp://ftp.ncbi. 
nih.gov/genomes/). The homologous map and proteome sequences of 417 core species were downloaded from eggNOG 3.0 [55].

\section{Features collection}

We collected 16 features (Table 1), 12 of which were widely used in previous models and 4 of which were used for the first time in our model (see details for the 16 features in Additional file 4: Table S6). The distribution difference of each feature between essential and non-essential genes is shown in Additional file 4: Figures S2-S13.

(i) Domain and GO annotations. Essential genes are associated with basic categories of biological functions or processes [52]. Therefore, essential genes may enrich some domains or GO annotations. To collect the domain of each gene in 21 species, the hidden Markov models (Pfam-A. $\mathrm{hmm}$ ) of the protein domains were downloaded from the Pfam database [56], and Hammer [57] was used to identify the protein domain for each gene. The corresponding domain type for each gene (see details of identifed domains for the 21 species in Additional file 5: Table S7) was defined as the feature DoT. The amino acid sites within protein domains are often more important and conserved than other fractions. Therefore, we assumed that the protein domain conservation is a reflection of gene essentiality, and the DoC of each gene was calculated from the ratio of the conserved domain score and the domain length. GO annotations were downloaded from the Gene Ontology Database [58]. GO enrichment analysis of SCE and $S P O$ is shown in Additional file 5: Table S8.

(ii) Protein-protein interaction (PPI) network. Network topology features have been widely used in previous papers (Additional file 4: Table S6). In our study, PPI data for the genes in 21 species were downloaded from the STRING Database [59]. Afterward, we used the NetworkX software package [60] to compute the four network topology features of DC, CCo, CC, and BC.

(iii) Genomic sequence properties. Although protein length (PL) tends to become longer through evolution [61], different natural constraints might exist on the PL between essential genes and nonessential genes. The codon usage of essential genes suffers from more evolutionary constraints than non-essential genes. We used the CodonW [62] software package to calculate the codon usage, i.e., CAI.

(iv) Homology properties. Duplicated genes are believed to often overlap in function and expression [63], and duplicates are always less likely to be essential than singletons $[34,64,65]$. An all-against-all BLAST search was conducted for the whole set of proteins in each of 21 species to identify the paralogs with an E-value threshold of $10^{-20}$, and the number of paralogs for a target gene within species was used as the feature NP. Four-hundred seventeen core organisms in the eggNOG database included all of 21 species in our study; thus, we counted the number of species among the 417 core species that had at least one homologous gene for each target gene in 21 species (feature NS). The orthologous gene of an essential gene is highly likely to be essential as well [18]. Therefore, we calculated the numbers of essential and non-essential homologous genes, including those that are found in other species, for each target gene (NEH and $\mathrm{NNH}$ ).

(v) Phyletic gene age. Chen [34] showed that older genes (i.e., genes with earlier phyletic origin) are more likely to be essential than young ones. Age was calculated according to previously described methods $[34,66]$; the target genomes of SCE and SPO were divided into five taxonomic groups, i.e., species typical, Ascomycota, Opisthokonta, Eukaryota, and cellular organisms.

(vi) Gene expression. mRNA expression data were obtained from Series GSE15352 [67] and GSE30025 [68] of the Gene Expression Omnibus (GEO) Database. The expression levels of essential genes are often generally higher and more stable than those of non-essential genes [69]. The average and variable coefficients of mRNA expression levels in all conditions were collected as predictors (i.e., $\mathrm{mE}$ and $\mathrm{mEF}$ ).

\section{Calculation of the feature score vector $S_{i j}$}

Features can be classified into two types: continuous and non-continuous. For continuous features, the kernel density estimation [KDE; the estimate is implemented by MATLAB's "ksdensity" function, using a normal kernel function and a window parameter (bandwidth) that is a function of the number of points in the sample] [49] is employed to acquire the empirical probability density function $f(x \mid E)$ for essential genes and $f(x \mid \bar{E})$ for nonessential genes [49]. The feature score vector $S_{i j}$ can be calculated as $S_{i j}=\log \frac{f(x \mid E)}{f(x \mid E)}$. For non-continuous features, the analysis is much more complicated (see Additional file 6). In the current study, we only displayed the inferred result: $S_{i j}=\log \frac{\left(n_{j E}+1\right) /\left(N_{E}+2\right)}{\left(n_{j \bar{E}}+1\right) /(N \bar{E}+2)}$, where $n_{j E}$ and $n_{j \bar{E}}$ indicate the number of essential and non-essential genes, respectively, sharing the same value for a given feature, and $N_{E}$ and $N \bar{E}$ indicate the total number of essential and nonessential genes, respectively.

\section{Other classifiers}

We compared three classifiers with FWM: (1) NBM, (2) LRM, and (3) SVM. Each classifier scheme independently generates a separate probability score of gene essentiality. All classifiers were implemented using the Weka software package [70]. The outline of Weka procedures with JAVA codes is shown in below.

Input: Attribute relation function format (ARFF) files of feature data of 21 organisms. 


\section{Parameters:}

1. Sequential minimal optimization (SMO) (weka. classifiers.functions. SMO -C 1.0 -L 0.001 -P 1.0E$12-\mathrm{N} 0-\mathrm{M}-\mathrm{V}-1-\mathrm{W} 1-\mathrm{K}$ "weka.classifiers.functions. supportVector.RBFKernel -C 250007 -G 0.01”)

2. NaïveBayes (default settings)

3. Logistic (weka.classifiers.functions. Logistic R 1.0E-8 -M -1)

Output: $21 \times 21$ AUC matrices of SVM, NBM, and LRM

1. Read the training and testing sets

2. For each species as training set

2.1.Build classifiers NaiveBayes(), Logistic() and $\mathrm{SMO}()$

2.2. For each species as testing set

2.2.1. Evaluate each classifier

2.2.2.Write evaluation.toSummaryString(), evaluation. toClassDetailsString(), evaluation. toMatrixString()2.2.3 Extract ROC area score

3. For each classifier

3.1. Arrange ROC area scores to the matrix

\section{Appendix: The derivation of formula (1) for FWM construction}

In Naïve Bayes algorithm, the probability that gene $g_{i}(i=1,2, \cdots, m)$ belongs to class $E(E=$ essential and $\bar{E}=$ non-essential $)$ given the feature vector $X_{i}=\left[x_{i 1}\right.$, $\left.x_{i 2}, \cdots, x_{i n}\right]$ is:

$$
P\left(g_{i} \in E \mid X_{i}\right)=\frac{P(E) \prod_{j=1}^{n} P\left(x_{i j} \mid g_{i} \in E\right)}{P\left(X_{i}\right)}
$$

where $j$ indicates the $j$ th feature in all $n$ features for gene $\left(g_{i}\right) ; P(E)$ indicates the prior probability of a gene belonging to an essential gene (in general, $P(E)$ is represented by the proportion of essential genes in all genes); $P\left(x_{i j} \mid g_{i} \in E\right)$ denotes the conditional probability when we observe that the $j$ th feature value is $x_{i j}$ under the condition that the $i$ th gene $\left(g_{i}\right)$ is an essential gene; and $P\left(X_{i}\right)$ is from the complete probability formula:

$$
P\left(X_{i}\right)=P(E) \prod_{j=1}^{n} P\left(x_{i j} \mid g i \in E\right)+P(\bar{E}) \prod_{j=1}^{n} P\left(x_{i j} \mid g_{i} \in \bar{E}\right)
$$

We obtain:

$$
P\left(g_{i} \in \bar{E} \mid X_{i}\right)=\frac{P(\bar{E}) \prod_{j=1}^{n} P\left(x_{i j} \mid g_{i} \in \bar{E}\right)}{P\left(X_{i}\right)}
$$

We use the ratio of (2) and (3) and set:

$$
P\left(g_{i} \in \bar{E} \mid X_{i}\right)=1-P\left(g_{i} \in E \mid X_{i}\right), P(\bar{E})=1-P(E)
$$

Finally, we obtain:

$$
P\left(g_{i} \in E \mid X_{i}\right)=\left[1+e^{-\log \frac{P(E)}{1-P(E)}-\sum_{j=1}^{n} S_{i j}}\right]^{-1},
$$

where $\frac{S_{i j}=\log P\left(x_{i j} \mid g_{i} \in E\right)}{P\left(x_{i j} \mid g_{i} \in \bar{E}\right)}$ indicates the logarithmic likelihood ratio, which we refer to as the feature score. We define the $G F S=\sum_{j=1}^{n} S_{i j}$ as the global feature score. If we suppose that GFS is a function of the feature vector $X_{i}$, the Naïve Bayes classifier comes into existence based on the fundamental conditions that the features must be mutually independent and that the training and prediction sets must have the same GFS function.

Unfortunately, the assumption of mutual independence for NBM does not always hold true, and training and prediction sets will not always have the same GFS function. To solve the problems, we add a weighted coefficient $w_{j}$ prior to $S_{i j}$. The global feature score is redefined as $G F S=\sum_{j=1}^{n} w_{j} S_{i j}$, and

$$
P\left(g_{i} \in E \mid X_{i}\right)=\left[1+e^{-\log _{1-P(E)} \frac{P(E)}{j-(E} \sum_{j=1}^{n}\left(w_{j} S_{i j}\right)}\right]^{-1}
$$

where $w_{j}$ indicates the extent of the contribution of the $j$ th feature to a gene classified as an essential gene. To simplify the model, we set $w_{0}=\log \frac{P(E)}{P(\bar{E})}, S_{i 0}=1, S_{i}=\left[S_{i 0}\right.$, $\left.S_{i 1}, S_{i 2}, \cdots, S_{i n}\right]$ and $W=\left[w_{0}, w_{1}, w_{2}, \cdots, w_{n}\right]$. The probability of a gene $g_{i}$ belonging to essential gene is given by:

$$
P\left(g_{i} \in E \mid X_{i}\right)=\frac{1}{1+e^{-S_{i} \cdot W}} .
$$

We put the scripts for FWM construction and usage in Additional file 7.

\section{Additional files}

Additional file 1: Figure S1. Essential gene prediction within and between species by NBM and FWM. AUC distributions within species (SPO - SPO) were generated by randomly selecting 20\% (A), 50\% (C) and 80\% (E) of SPO genes as training data and training-prediction set, respectively. Whereas AUC distributions between species (SPO- SCE) were generated by randomly selecting 20\% (B), 50\% (D), and 80\% (F) of SCE genes as trainingprediction set to estimate weight vector $W$, respectively. The blue and red lines represent the distribution obtained by NBM and FWM, respectively.

Additional file 2: Table S1. The fundamental information of 21 species. This table displayed the evolutionary relationships of 21 species, the experimental methods for identification of essential genes, and the growth conditions. Table S2. The AUC matrices of four methods. Each matrix was obtained with the corresponding method. The AUC score in the same position of matrices had the same training set and prediction set. Table S3. The AUC order matrices of four methods. The AUC scores $\left(m_{i j}\right)$ in the same position of the four matrices were sorted and replaced with numbers ( 1 was equivalent to the maximum AUC score, followed by 
2 and 3 , and 4 corresponded to the minimum score). We then calculated the frequency of the ranking list (i.e., 1, 2, 3, and 4) in the four matrices. The method which the corresponding matrix had more 1 or 2 was considered better method. Table S4. The AUC scores with singular feature in the predictions. Table S5. Comparing FWM and NBM in stepwise discriminant analysis. We employed SDA to investigate AUC variations by adding features one by one. The 'Features' column indicates the sequence into the model. The 'Singular AUC Score' column indicates the AUC score generated with only a feature in the model. The 'Ranking' column indicates the sorting of singular AUC score. The ' $W$ ' column indicates the weights of the corresponding feature in FWM. The last two columns indicate AUC variations with the additions of features).

Additional file 3: Essential genes dataset. We collected currently available essential genes among a wide range of organisms, including 11 eukaryotes and 21 prokaryotes.

Additional file 4: Table S6. Introduction and reference about our selected features. Figure S2. The distribution difference of DoC. Figure S3. The distribution difference of DC. Figure S4. The distribution difference of CCo. Figure S5. The distribution difference of CC. Figure S6. The distribution difference of $\mathrm{BC}$. Figure S7. The distribution difference of PL. Figure S8. The distribution difference of CAl. Figure S9. The distribution difference of NP. Figure S10. The distribution difference of NS. Figure S11. The distribution difference of NEH. Figure S12. The distribution difference of NNH. Figure S13. The distribution difference of $\mathrm{mE}, \mathrm{mEF}$, and Age in SCE and SPO.

Additional file 5: Table S7. Comparison of domain enrichment between the 19 bacteria and 2 fungi. We calculate the P-value using the hypergeometric distribution, and Bonferroni method is used for multiple hypothesis correction. Table S8. Comparison of Gene Ontology term enrichment between S. cerevisiae and S. pombe. We calculate the P-value using the hypergeometric distribution, and Bonferroni method is used for multiple hypothesis correction.

Additional file 6: Calculation of the score vector $S_{i}$ for noncontinuous features.

Additional file 7: FWM script.

\section{Competing interests}

The authors declare that they have no competing interests.

\section{Authors' contributions}

JC conceived and designed the method. JC and WW performed the analyses. WW wrote the manuscript. All authors have read and approve of the final manuscript.

\section{Acknowledgments}

We are grateful to the members of the Bioinformatics Center of Northwest A\&F University for providing many useful suggestions. WWW's Research is supported by China Postdoctoral Science Foundation funded project (2013M541564).

\section{Author details}

${ }^{1}$ College of Life Science, State Key Laboratory of Crop Stress Biology for Arid Areas, Northwest A\&F University, Yangling, Shaanxi, China. ${ }^{2}$ Bioinformatics Center, Northwest A\&F University, Yangling 712100, Shaanxi, China. ${ }^{3}$ Key Laboratory of Food Safety Research, Institute for Nutritional Sciences, Shanghai Institutes for Biological Sciences, Chinese Academy of Sciences, University of Chinese Academy of Sciences, Shanghai 200031, China. ${ }^{4}$ College of Life Science, Northwest A\&F University, Yangling 712100, Shaanxi, China. ${ }^{5}$ College of Science, Northwest A\&F University, Yangling 712100, Shaanxi, China.

Received: 15 June 2013 Accepted: 29 November 2013

Published: 21 December 2013

\section{References}

1. Itaya M: An estimation of minimal genome size required for life. FEBS letters 1995, 362(3):257-260.
2. Kobayashi K, Ehrlich SD, Albertini A, Amati G, Andersen K, Arnaud M, Asai K, Ashikaga S, Aymerich S, Bessieres P: Essential Bacillus subtilis genes. Proc Natl Acad Sci 2003, 100(8):4678-4683.

3. Papp B, Pal C, Hurst LD: Metabolic network analysis of the causes and evolution of enzyme dispensability in yeast. Nature 2004, 429(6992):661-664.

4. Yu H, Greenbaum D, Lu HX, Zhu X, Gerstein M: Genomic analysis of essentiality within protein networks. RNA 2004, 71:817-846.

5. Gerdes S, Edwards R, Kubal M, Fonstein M, Stevens R, Osterman A: Essential genes on metabolic maps. Curr Opin Biotechnol 2006, 17(5):448.

6. D'Elia MA, Pereira MP, Brown ED: Are essential genes really essential? Trends Microbiol 2009, 17(10):433-438.

7. Dowell RD, Ryan O, Jansen A, Cheung D, Agarwala S, Danford T, Bernstein DA, Rolfe PA, Heisler LE, Chin B: Genotype to phenotype: a complex problem. Science 2010, 328(5977):469-469.

8. Dickerson JE, Zhu A, Robertson DL, Hentges KE: Defining the role of essential genes in human disease. PloS one 2011, 6(11):e27368.

9. Chalker AF, Lunsford RD: Rational identification of new antibacterial drug targets that are essential for viability using a genomics-based approach. Pharmacol Ther 2002, 95(1):1-20.

10. Cole S: Comparative mycobacterial genomics as a tool for drug target and antigen discovery. Eur Respir J 2002, 20(36 suppl):78s-86s.

11. Gibson DG, Glass Jl, Lartigue C, Noskov VN, Chuang R-Y, Algire MA, Benders GA, Montague MG, Ma L, Moodie MM: Creation of a bacterial cell controlled by a chemically synthesized genome. Science 2010, 329(5987):52-56.

12. Re C, Bott T, El M, MIR U, Ifornia S, Dieg A: Synthetic genome brings new life to bacterium. Science 2007, 18:965.

13. Giaever G, Chu AM, Ni L, Connelly C, Riles L, V"'ronneau S, Dow S, Lucau-Danila A, Anderson K, Andr"' B: Functional profiling of the Saccharomyces cerevisiae genome. Nature 2002, 418(6896):387-391.

14. Roemer T, Jiang B, Davison J, Ketela T, Veillette K, Breton A, Tandia F, Linteau A, Sillaots S, Marta C: Large-scale essential gene identification in Candida albicans and applications to antifungal drug discovery. Mol Microbiol 2003, 50(1):167-181.

15. Cullen LM, Arndt GM: Genome-wide screening for gene function using RNAi in mammalian cells. Immunol Cell Biol 2005, 83(3):217-223.

16. Salama NR, Shepherd B, Falkow S: Global transposon mutagenesis and essential gene analysis of Helicobacter pylori. J Bacteriol 2004 186(23):7926-7935.

17. Fang G, Rocha E, Danchin A: How essential are nonessential genes? Mol Biol Evol 2005, 22(11):2147-2156.

18. Wang S, Sim TB, Kim Y-S, Chang Y-T: Tools for target identification and validation. Curr Opin Chem Biol 2004, 8(4):371-377.

19. Krasky A, Rohwer A, Schroeder J, Selzer P: A combined bioinformatics and chemoinformatics approach for the development of new antiparasitic drugs. Genomics 2007, 89(1):36-43.

20. Kumar S, Chaudhary K, Foster JM, Novelli JF, Zhang Y, Wang S, Spiro D, Ghedin E, Carlow CK: Mining predicted essential genes of Brugia malayi for nematode drug targets. PloS one 2007, 2(11):e1189.

21. Holman AG, Davis PJ, Foster JM, Carlow CKS, Kumar S: Computational prediction of essential genes in an unculturable endosymbiotic bacterium, Wolbachia of Brugia malayi. BMC Microbiol 2009, 9(1):243.

22. Bruccoleri RE, Dougherty TJ, Davison DB: Concordance analysis of microbial genomes. Nucleic Acids Res 1998, 26(19):4482-4486

23. Kim DU, Hayles J, Kim D, Wood V, Park HO, Won M, Yoo HS, Duhig T, Nam M, Palmer G: Analysis of a genome-wide set of gene deletions in the fission yeast Schizosaccharomyces pombe. Nat Biotechnol 2010, 28(6):617-623

24. Seringhaus M, Paccanaro A, Borneman A, Snyder M, Gerstein M: Predicting essential genes in fungal genomes. Genome Res 2006, 16(9):1126.

25. Gustafson AM, Snitkin ES, Parker SCJ, DeLisi C, Kasif S: Towards the identification of essential genes using targeted genome sequencing and comparative analysis. Bmc Genomics 2006, 7(1):265.

26. Acencio ML, Lemke N: Towards the prediction of essential genes by integration of network topology, cellular localization and biological process information. BMC Bioinformatics 2009, 10(1):290.

27. Plaimas $K$, Eils $R$, König R: Identifying essential genes in bacterial metabolic networks with machine learning methods. BMC Syst Biol 2010, 4(1):56. 
28. Deng J, Deng L, Su S, Zhang M, Lin X, Wei L, Minai AA, Hassett DJ, Lu LJ: Investigating the predictability of essential genes across distantly related organisms using an integrative approach. Nucleic Acids Res 2011, 39(3):795.

29. Pržulj N, Wigle $D$, Jurisica I: Functional topology in a network of protein interactions. Bioinformatics 2004, 20(3):340-348.

30. Hahn MW, Kern AD: Comparative genomics of centrality and essentiality in three eukaryotic protein-interaction networks. Mol Biol Evol 2005, 22(4):803-806

31. Gursoy A, Keskin O, Nussinov R: Topological properties of protein interaction networks from a structural perspective. Biochem Soc Trans 2008, 36(6):1398

32. Saeys $Y$, Inza I, Larrañaga $P$ : A review of feature selection techniques in bioinformatics. Bioinformatics 2007, 23(19):2507-2517.

33. Chen S, Zhang YE, Long M: New genes in Drosophila quickly become essential. Science 2010, 330(6011):1682-1685.

34. Chen W-H, Trachana K, Lercher MJ, Bork P: Younger genes are less likely to be essential than older genes, and duplicates are less likely to be essentia than singletons of the same age. Mol Biol Evol 2012, 29(7):1703-1706.

35. Liao B-Y, Zhang J: Mouse duplicate genes are as essential as singletons. Trends Genet 2007, 23(8):378-381.

36. Makino T, Hokamp K, McLysaght A: The complex relationship of gene duplication and essentiality. Trends Genet 2009, 25(4):152-155.

37. Glodberg DE: Genetic algorithms in search, optimization, and machine learning Boston, MA, USA: Addison-Wesley Longman Publishing Co., Inc:; 1989.

38. Frank E, Hall M, Pfahringer B: Locally weighted naive bayes. Morgan Kaufmann Publishers Inc; 2002:249-256.

39. Myers RH: Classical and modern regression with applications, vol. 2. Belmont, CA: Duxbury Press; 1990

40. Hocking RR: A Biometrics invited paper. The analysis and selection of variables in linear regression. Biometrics 1976, 32(1):1-49.

41. Draper NR, Smith H, Pownell E: Applied regression analysis, vol. 3. New York: Wiley; 1966

42. Price MN, Deutschbauer AM, Skerker JM, Wetmore KM, Ruths T, Mar JS Kuehl JV, Shao W, Arkin AP: Indirect and Suboptimal Control of Gene Expression is Widespread in Bacteria. Molecular systems biology 2013, 9(1).

43. Steinmetz LM, Scharfe C, Deutschbauer AM, Mokranjac D, Herman ZS, Jones T, Chu AM, Giaever G, Prokisch H, Oefner PJ: Systematic screen for human disease genes in yeast. Nat Gen 2002, 31(4):400-404

44. Yamazaki Y, Niki H, Kato J: Profiling of Escherichia coli Chromosome database. Methods Mol Biol 2008, 416:385-389.

45. Rish I: An empirical study of the naive Bayes classifier, IJCAI 2001 workshop on empirical methods in artificial intelligence: 2001. 2001:41-46.

46. De Ferrari L, Aitken S: Mining housekeeping genes with a Naive Bayes classifier. Bmc Genomics 2006, 7(1):277.

47. Calvo S, Jain M, Xie X, Sheth SA, Chang B, Goldberger OA, Spinazzola A, Zeviani M, Carr SA, Mootha VK: Systematic identification of human mitochondrial disease genes through integrative genomics. Nat Gen 2006, 38(5):576-582.

48. Lee I, Blom UM, Wang PI, Shim JE, Marcotte EM: Prioritizing candidate disease genes by network-based boosting of genome-wide association data. Genome Res 2011, 21(7):1109-1121.

49. Terrell GR, Scott DW: Variable kernel density estimation. Annals Stat 1992 20(3):1236-1265

50. Lachenbruch PA, Goldstein M: Discriminant analysis. Biometrics 1979 35(1):69-85

51. Fawcett T: ROC graphs: notes and practical considerations for researchers. Mach Learn 2004, 31:1-38.

52. Xu P, Ge X, Chen L, Wang X, Dou Y, Xu JZ, Patel JR, Stone V, Evans K, Kitten $T$ : Genome-wide essential gene identification in Streptococcus sanguinis. Sci Rep 2011, 1:125.

53. Chen W-H, Minguez P, Lercher MJ, Bork P: OGEE: an online gene essentiality database. Nucleic Acids Res 2012, 40(D1):D901-D906.

54. Zhang R, Ou HY, Zhang CT: DEG: a database of essential genes. Nucleic Acids Res 2004, 32(suppl 1):D271.

55. Powell S, Szklarczyk D, Trachana K, Roth A, Kuhn M, Muller J, Arnold R, Rattei T, Letunic I, Doerks T: eggNOG v3. 0: orthologous groups covering 1133 organisms at 41 different taxonomic ranges. Nucleic Acids Res 2012, 40(D1):D284-D289.
56. Punta M, Coggill PC, Eberhardt RY, Mistry J, Tate J, Boursnell C, Pang N, Forslund K, Ceric G, Clements J: The Pfam protein families database. Nucleic Acids Res 2012, 40(D1):D290-D301.

57. Finn RD, Clements J, Eddy SR: HMMER web server: interactive sequence similarity searching. Nucleic Acids Res 2011, 39(suppl 2):W29-W37.

58. Ashburner M, Ball CA, Blake JA, Botstein D, Butler H, Cherry JM, Davis AP, Dolinski K, Dwight SS, Eppig JT: Gene Ontology: tool for the unification of biology. Nat Gen 2000, 25(1):25-29.

59. Szklarczyk D, Franceschini A, Kuhn M, Simonovic M, Roth A, Minguez $P$, Doerks T, Stark M, Muller J, Bork P: The STRING database in 2011: functional interaction networks of proteins, globally integrated and scored. Nucleic Acids Res 2011, 39(suppl 1):D561-D568.

60. Hagberg A, Swart P, Shult D: Exploring network structure, dynamics, and function using networkX. In Proceedings of the 7th Python in Science conference (SciPy 2008). Edited by Varoquaux G, Vaught T, Millman J. :11-15.

61. Lipman DJ, Souvorov A, Koonin EV, Panchenko AR, Tatusova TA: The relationship of protein conservation and sequence length. BMC Evol Biol 2002, 2(1):20.

62. Peden JF: Analysis of codon usage. In PhD Thesis. UK: University of Nottingham; 1999

63. Ohno S: Evolution by gene duplication: London: George Alien \& Unwin Ltd. Berlin, Heidelberg and New York: Springer; 1970

64. Gu Z, Steinmetz L, Gu X, Scharfe C, Davis R, Li W: Role of duplicate genes in genetic robustness against null mutations. Nature 2003, 421(6918):63-66.

65. Woods S, Coghlan A, Rivers D, Warnecke T, Jeffries SJ, Kwon T, Rogers A, Hurst LD, Ahringer J: Duplication and retention biases of essential and Non-essential genes revealed by systematic knockdown analyses. PLoS genetics 2013, 9(5):e1003330

66. Wolf YI, Novichkov PS, Karev GP, Koonin EV, Lipman DJ: The universal distribution of evolutionary rates of genes and distinct characteristics of eukaryotic genes of different apparent ages. Proc Natl Acad Sci 2009, 106(18):7273-7280.

67. Strassburg K, Walther D, Takahashi H, Kanaya S, Kopka J: Dynamic transcriptional and metabolic responses in yeast adapting to temperature stress. OMICS 2010, 14(3):249-259.

68. Jia X, He W, Murchie Al, Chen D: The Global Transcriptional Response of Fission Yeast to Hydrogen Sulfide. PloS one 2011, 6(12):e28275.

69. Jeong H, Oltvai ZN, Barabási A-L: Prediction of protein essentiality based on genomic data. ComplexUs 2002, 1(1):19-28.

70. Hall M, Frank E, Holmes G, Pfahringer B, Reutemann P, Witten $I H$ : The WEKA data mining software: an update. ACM SIGKDD Explorations Newsletter 2009, 11(1):10-18.

doi:10.1186/1471-2164-14-910

Cite this article as: Cheng et al: A new computational strategy for predicting essential genes. BMC Genomics 2013 14:910.

\section{Submit your next manuscript to BioMed Central and take full advantage of:}

- Convenient online submission

- Thorough peer review

- No space constraints or color figure charges

- Immediate publication on acceptance

- Inclusion in PubMed, CAS, Scopus and Google Scholar

- Research which is freely available for redistribution 